J. Dairy Sci. 92:943-951

doi:10.3168/jds.2008-1548

(c) American Dairy Science Association, 2009.

\title{
Epidemiological investigation of Streptococcus equi subspecies zooepidemicus involved in clinical mastitis in dairy goats
}

\author{
G. Pisoni, ${ }^{*}$ R. N. Zadoks,$\dagger^{1}$ C. Vimercati, ${ }^{*}$ C. Locatelli, ${ }^{*}$ M. G. Zanoni, $\neq$ and P. Moroni ${ }^{* 2}$ \\ *Department of Veterinary Pathology, Hygiene and Public Health, University of Milan, 20133 Milan, Italy \\ †Quality Milk Production Services, Cornell University, Ithaca, NY 14850-1263 \\ łlstituto Zooprofilattico Sperimentale della Lombardia e dell'Emilia Romagna, 25124 Brescia, Italy
}

\section{ABSTRACT}

An outbreak of clinical mastitis was observed in dairy goats due to the zoonotic pathogen Streptococcus equi ssp. zooepidemicus. Affected goats were culled to prevent transmission of infection to other animals or humans. The objective of the study was to determine whether horses on the same farm were the source of the pathogen. Streptococcus equi ssp. zooepidemicus was obtained from milk of $10 \%$ of goats in the herd and from feces of 3 of 7 healthy horses that shared pasture and housing with the goats. Isolates of caprine and equine origin had identical biochemical profiles, including the ability to ferment sorbitol and lactose, which distinguishes $S$. equi ssp. zooepidemicus from $S$. equi ssp. equi. Sequencing of the $16 \mathrm{~S}-23 \mathrm{~S}$ intergenic spacer region and results from sodA-seeI multiplex PCR supported identification of isolates as S. equi ssp. zooepidemicus. Based on random amplified polymorphic DNA typing and rpoB and $\operatorname{sod} A$ sequencing, caprine isolates were indistinguishable from each other, but distinct from equine isolates. Further analysis of equine fecal samples showed that multiple strains of $S$. equi ssp. zooepidemicus can be present in a single sample or in sequential samples obtained from a single horse. Failure to detect the mastitis-causing strain in equine feces may indicate that horses were not the source of the mastitis outbreak in goats. Alternatively, the outbreak may be due to presence of multiple $S$. equi ssp. zooepidemicus strains in equine feces and a failure to detect all strains when analyzing a limited number of isolates per sample.

Key words: Streptococcus equi ssp. zooepidemicus, goat, horse, mastitis

Received July 14, 2008.

Accepted October 20, 2008

${ }^{1}$ Current address: Moredun Research Institute, Pentlands Science Park, Bush Loan, Midlothian, EH26 0PZ, UK.

${ }^{2}$ Corresponding author: paolo.moroni@unimi.it

\section{INTRODUCTION}

Streptococcus equi ssp. equi (SEE) and Streptococcus equi ssp. zooepidemicus (SEZ) are $\beta$-hemolytic group C Streptococcus species that cause disease in animals and humans. Phylogenetically, SEZ is regarded as the ancestral species of the closely related clonal subspecies SEE (Chanter et al., 1997; Harrington et al., 2002; Timoney, 2004). Identification and differentiation of subspecies was traditionally based on biochemical reactions such as hydrolysis of esculin, fermentation of sorbitol, and fermentation of trehalose (Harrington et al., 2002). Recent developments in nucleic acid technology resulted in new methods for differentiation of the 2 subspecies, such as PCR-mediated identification of species-specific segments of the 16S-23S rDNA intergenic spacer regions (ISR; Chanter et al., 1997; Hassan et al., 2003). Streptococcus equi ssp. zooepidemicus is commonly found colonizing the mucous membranes of healthy equids. It is associated with respiratory tract infections in foals and with uterine infections in mares (Timoney, 2004). Moreover, SEZ is a possible cause of mastitis in dairy ruminants (Sharp et al., 1995; Las Heras et al., 2002). The IMI caused by SEZ were named pseudo-agalactia because the clinical signs were similar to those observed in chronic contagious agalactia due to Mycoplasma spp. Previous studies described cases of clinical mastitis in dairy ruminants and hypothesized the role of equids as the origin of infections (Sharp et al., 1995; Bezek, 1998; Las Heras et al., 2002). None of the studies cited employed molecular typing techniques to compare SEZ strains involved in mastitis outbreaks to isolates from other host species. Unlike SEE, SEZ is found in human infections. Human infections are rare and the clinical presentations include pharyngitis, septicemia, meningitis, purulent arthritis, and endocarditis (Barnham et al., 1983; Balter et al., 2000). The source of human infection was often traced back to contact with domestic animals, especially horses, or ingestion of unpasteurized milk or milk products (Edwards et al., 1988; Bordes-Benítez et al., 2006; Kuusi et al., 2006). For example, this organism was associated with a large 
outbreak of nephritis in Brazil that was attributed to consumption of unpasteurized cheese (Balter et al., 2000).

An outbreak of SEZ mastitis is described in a goat herd that produced milk for the manufacture of raw milk cheese. Based on molecular typing, horses that were present on the same farm as the goats were investigated as the probable source of infection. Methodological aspects of this case are discussed, and suggestions for improvement of methodology in future outbreak analysis are made.

\section{MATERIALS AND METHODS}

\section{Case History}

A herd of goats on a small-scale commercial dairy farm in northern Italy (Varese, Lombardy) was monitored monthly throughout lactation (i.e., from March to September) for milk quality and production traits. Technicians of the Provincial Breeding Association of Varese (Lombardy) collected milk samples for measurement of SCC, fat and protein content, and milk yield. In addition, milk samples were collected aseptically from each udder half for routine bacteriological analysis. The herd grazed on natural pastures for at least $8 \mathrm{~h}$ daily. Animals were housed in a barn during the night. The ration was supplemented with concentrates, and water was available at all times. The farm practiced seasonal milking and the does kidded between January and February. After weaning, all goats were hand-milked twice daily inside the barn by the same milker without teat preparation or cleaning. Milk was taken in a plastic box for daily milk yield measurement, collected in a bucket, and then stored in a refrigerated bulk tank. Milk was used to manufacture raw cheeses. Teat disinfection and application of antimicrobials during the dry period were not practiced. The farm was free of brucellosis and mycoplasmosis. The goats had no contact with other dairy ruminants, but they shared pastures and a barn with 7 horses.

At the end of May, the farmer observed the appearance of clinical signs of mastitis in 2 of 22 lactating goats. Mammary glands appeared swollen, firm, and painful at palpation, and the milk contained small clots. Daily milk production decreased in the affected mammary glands, which dried up 2 to $3 \mathrm{~d}$ after onset of symptoms. Affected goats showed systemic signs of illness (fever, anorexia, depression, and decreased rumen motility). Infected animals were segregated from the herd as a preventive measure to avoid goat-to-goat transmission. No goats died, but the affected animals were prematurely slaughtered as soon as the bacteriological diagnosis was known because of the contagious and zoonotic nature of the pathogen. No further cases of clinical mastitis were observed in the herd during the remainder of the lactation.

\section{Milk Sample Processing}

Milk samples were collected aseptically from individual half-udders of all goats. Teat ends were cleaned and disinfected with chlorhexidine before sampling. The first streams of foremilk were discarded, and $10 \mathrm{~mL}$ of milk was collected into sterile vials. Samples were kept under refrigeration until bacteriological analysis was performed using standard methods (National Mastitis Council, 1999). Briefly, $10 \mu \mathrm{L}$ of each milk sample was spread on blood agar plates $(5 \%$ defibrinated sheep blood, Microbiol Diagnostici, Cagliari, Italy). Plates were incubated aerobically at $37^{\circ} \mathrm{C}$ and examined after $24 \mathrm{~h}$. $\beta$-Hemolytic colonies were provisionally identified as streptococci based on Gram stain, morphology, and catalase reaction. The Christie-Atkins-Munch-Petersen (CAMP) test was performed using conventional procedures (National Mastitis Council, 1999). The isolates were further characterized biochemically using commercial methods (API 20 Strep, BioMérieux, Rome, Italy) following the instructions of the manufacturer.

\section{Fecal and Nasal Sample Processing}

Fecal samples were collected using single-use plastic gloves from the rectum of 7 horses that shared the same pastures and barn as the goats. Samples were kept at $4^{\circ} \mathrm{C}$ and processed within $24 \mathrm{~h}$ of collection. For detection of presumptive Streptococcus spp., $1 \mathrm{~g}$ of fecal matter was suspended in $9 \mathrm{~mL}$ of sterile buffered peptone saline solution (Merck KGaA, Darmstadt, Germany) and $10 \mu \mathrm{L}$ of the suspension was spread on blood agar plates.

A single nasal swab was collected from each horse. A cotton-tipped culture swab (BD Diagnostic Systems, Milan, Italy) was inserted approximately $10 \mathrm{~cm}$ into 1 nasal passage and withdrawn with the swab in contact with the nasal mucosa. Swabs were promptly placed in transport medium (Cultiplast with Amies, LP Italiana, Milan, Italy) and kept at $4^{\circ} \mathrm{C}$ until processing. Swabs were placed in $2 \mathrm{~mL}$ of enrichment broth consisting of $10 \mathrm{~g} / \mathrm{L}$ of tryptone $\mathrm{T}, 75 \mathrm{~g} / \mathrm{L}$ of sodium chloride, and $2.5 \mathrm{~g} / \mathrm{L}$ of yeast extract (Merck KGaA) and incubated aerobically at $37^{\circ} \mathrm{C}$ for $24 \mathrm{~h}$. Approximately $100 \mu \mathrm{L}$ of broth was then inoculated onto blood agar plates.

Inoculated blood agar plates were incubated aerobically at $37^{\circ} \mathrm{C}$ and examined after $24 \mathrm{~h}$. Isolates were subsequently processed as described for milk samples. If a horse tested positive for SEZ in a fecal sample, further fecal samples were collected at subsequent herd 
Table 1. Targets, primers, and cycling measures used for characterization of Streptococcus equi ssp. zooepidemicus isolates from goats and horses

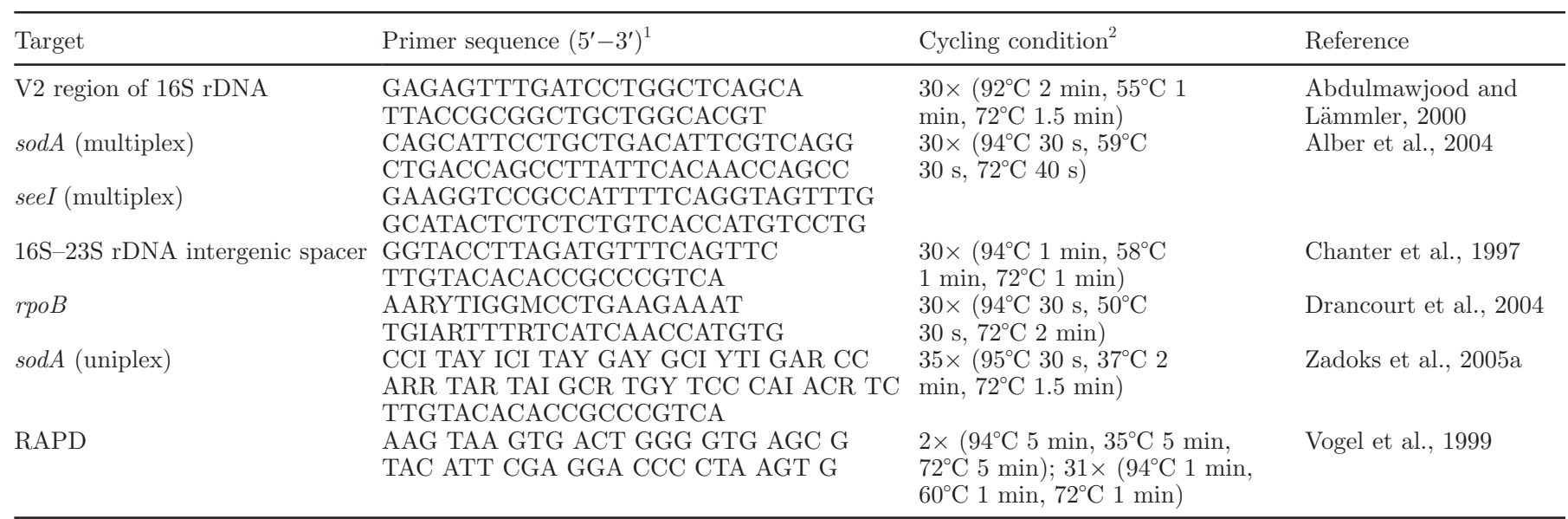

${ }^{1}$ First primer shown is forward primer, second primer shown is reverse primer, with the exception of primers shown for random amplified polymorphic DNA (RAPD; first primer: ERIC-2, second primer: ERIC1026).

${ }^{2}$ All PCR programs started with an initial 4-min denaturation step at $94^{\circ} \mathrm{C}$ and ended with a final 7 -min elongation step at $72^{\circ} \mathrm{C}$.

visits (after $1 \mathrm{wk}, 1 \mathrm{mo}$, and $6 \mathrm{mo}$ ) to obtain additional isolates representative of the gastrointestinal flora of each animal. To obtain single colonies, $1 \mathrm{~g}$ of fecal matter was suspended in $9 \mathrm{~mL}$ of sterile buffered peptone saline solution and serial 10-fold dilutions were prepared. One hundred microliters of dilution $10^{-1}, 10^{-2}$, $10^{-3}$, and $10^{-4}$ were streaked on blood agar plates for single colony isolation. The plates were incubated aerobically at $37^{\circ} \mathrm{C}$ and examined after $24 \mathrm{~h}$. For each fecal sample, 10 isolated colonies were selected and used for subsequent molecular analysis. The majority of isolates were derived from the $10^{-2}$ and $10^{-3}$ dilution.

\section{Molecular Typing}

For molecular assays, pure subcultures obtained from single colonies of SEZ identified during bacteriology procedures were frozen and stored at $-70^{\circ} \mathrm{C}$ in a nutrient broth $(5 \mathrm{~g} / \mathrm{L}$ of tryptone, $3 \mathrm{~g} / \mathrm{L}$ of meat extract, Merck KGaA) enriched with 15\% of glycerol until DNA extraction could be performed. Nucleic acids were extracted from $1 \mathrm{~mL}$ of bacterial suspension using the Puregene DNA Purification Kit (Gentra Systems, Minneapolis, MN) according to the recommendation of the manufacturer for gram-positive bacteria.

To confirm species identity, isolates obtained from goats and horses at the initial sampling were characterized by amplification and sequencing of the V2 region of the 16S rRNA gene (Abdulmawjood and Lämmler, 2000), by sodA-seeI multiplex PCR (Alber et al., 2004), and by polymorphism analysis of the $16 \mathrm{~S}-23 \mathrm{~S}$ rDNA ISR (16S-23S ISR; Chanter et al., 1997; Alber et al., 2004; Younan et al., 2005). The housekeeping genes rpoB and $\operatorname{sodA}$ were amplified and sequenced (Drancourt et al., 2004; Zadoks et al., 2005a) to explore strain heterogeneity. In addition, random amplified polymorphic DNA typing (RAPD) was performed (Vogel et al., 1999), using SEE (ATCC 33398), SEZ (ATCC 700400), and Klebsiella pneumoniae (ATCC 13883) as positive control strains.

Species and strain level amplification targets, oligonucleotide primer sequences, and thermal cycling conditions are summarized in Table 1. Electrophoresis of amplified products was carried out using 1.5\% agarose gels (Promega, Milan, Italy) at $100 \mathrm{~V}$ and gels were stained with ethidium bromide (Euroclone, Celbio Spa, Milan, Italy) and visualized through UV transillumination. Amplification products of $16 \mathrm{~S}-23 \mathrm{~S}$ ISR, sodA, and rpoB PCR were purified with the QIAquick PCR Purification Kit (Qiagen, Milan, Italy; for 16S-23S ISR) or PureLink PCR Purification Kit (Invitrogen Corporation, Carlsbad, CA; for rpoB and sodA) and sequenced, with both PCR primers, by CRIBI Services (CRIBI, Padova, Italy; for 16S-23S ISR), or by the Biotechnology Resource Center (Cornell University, Ithaca, NY; for $\operatorname{rpo} B$ and $\operatorname{sod} A$ ), on an ABI377 sequencer by using the ABI PRISM dye-terminator cycle sequencing ready reaction kit with Amplitaq DNA polymerase (PerkinElmer, Applied Biosystems).

To assess strain diversity within fecal samples, RAPD was performed for between 10 and 20 isolates per fecal sample from each animal, using the same primers and cycling conditions as used for comparison of isolates from milk and feces (Table 1) and the same control strains. Fecal isolates were compared with the milk isolates obtained from goats. For accurate comparison, 
all SEZ isolates included in a comparison were grown, purified, amplified, and run on a gel in the same batch. Isolates with banding patterns with the same number and size of DNA fragments were considered to belong to the same strain, regardless of band intensity.

\section{RESULTS}

\section{Clinical Observations and Bacteriology}

The outbreak involved 2 of 22 lactating goats. Both goats (goats 922 and 928) showed local and systemic signs of clinical mastitis. Somatic cell counts were elevated $\left(>7 \times 10^{6} \mathrm{SCC} / \mathrm{mL}\right)$ in milk of both animals. The daily milk production of infected goats ranged between 900 and 1,500 $\mathrm{mL}$ before mastitis was diagnosed and decreased drastically in the affected mammary glands, which ceased milk production 2 to $3 \mathrm{~d}$ after the onset of symptoms. Pure cultures of $\beta$ hemolytic microorganisms, suspected to be SEZ, were isolated from the milk of goats 922 and 928 but not from milk of other goats in the same herd. Suspected SEZ colonies were detected in 3 of 7 fecal samples from healthy horses (horses $\mathrm{CV} / 2$, $\mathrm{CV} / 3$, and $\mathrm{CV} / 6$ ) but not in nasal swabs. Suspected SEZ colonies were detected in fecal samples from the 3 horses during subsequent samplings. All selected isolates from milk and feces were gram-positive, catalasenegative, CAMP (Christie-Atkins-Munch-Petersen)negative cocci. According to the API 20 Strep System, all isolates were identified as SEZ, which is differentiated from SEE based on the ability of SEZ, but not SEE, to ferment sorbitol and lactose. All caprine and equine SEZ isolates had identical biochemical profiles. The SEZ counts in fecal samples ranged from $1 \times 10^{5}$ to $2 \times 10^{6} \mathrm{cfu} / \mathrm{mL}$.

\section{Molecular Typing}

Based on sequence analysis of a 490-bp segment of the V2 region of the 16S rRNA gene, all caprine and equine isolates obtained belonged to $16 \mathrm{~S}$ rRNA type 1, which included SEE as well as SEZ isolates (Abdulmawjood and Lämmler, 2000). Complete sequence identity of the amplified V2 regions was observed for the 2 caprine isolates (GenBank accession number EU784820, V2 region of caprine milk isolates 922 and 928) and with the $\mathrm{V} 2$ regions of SEZ isolates Bd 2/04 and Bd 643/03 (Baverud et al., 2007). The V2 sequence for caprine isolates differed from the $\mathrm{V} 2$ sequence for the equine fecal isolates and SEE reference strains belonging to 16S rRNA type 1 (CCUG 27367 and ATCC 33398) by a single nucleotide. Among 3 isolates from equine feces, 2 V2 alleles were observed (EU784821, V2 region of equine fecal isolates $\mathrm{CV} / 2$ and $\mathrm{CV} / 6$, identical to
V2 sequence of SEE isolates CCUG 27367 and ATCC 33398; EU784822, V2 region of equine fecal isolate $\mathrm{CV} / 3$, identical to $\mathrm{V} 2$ sequence of SEZ isolates $\mathrm{Bd}$ 16498/05, 3613/03, 16000/02, and 13524/2; Baverud et al., 2007).

Identification of caprine and equine isolates obtained as SEZ was confirmed by sodA-seeI multiplex PCR. All isolates showed sodA gene amplification (235 bp), whereas they were negative to seeI gene amplification (520 bp) in multiplex PCR (Figure 1). Amplification of the 16S-23S ISR region yielded a single PCR amplicon with a size of approximately $780 \mathrm{bp}$ for each of the caprine $(\mathrm{n}=2)$ and equine $(\mathrm{n}=3)$ isolates, in agreement with identification of isolates as SEZ. Amplicon size for SEE is 950 bp (Hassan et al., 2003). The classification of the 16S-23S ISR of all isolates in this study (Table 2), performed according to Chanter et al. (1997) and Hassan et al. (2003), showed that our strains were more similar to SEZ than to SEE based on the lack of the R3 region typical of SEE strains, and the size of the $\mathrm{R} 8$ region (Figure 2).

Differentiation of isolates at the subspecies level was obtained through ISR, $\operatorname{rpo} B$, and $\operatorname{sod} A$ sequencing as well as RAPD typing. All methods showed that the 2 caprine isolates were indistinguishable from each other but distinct from the 3 equine fecal isolates obtained during the first fecal sampling. Regardless of the typing method used, equine isolates $\mathrm{CV} / 2$ and $\mathrm{CV} / 6$ were indistinguishable from each other but distinct from equine isolate $\mathrm{CV} / 3$. The ISR of the 2 isolates from goats revealed complete sequence identity (100\%). Intergenic spacer regions of isolates from horses $\mathrm{CV} / 2$ and $\mathrm{CV} / 6$ revealed complete sequence identity to each other and sequence identity of 98.6 and $81.2 \%$ with horse isolate $\mathrm{CV} / 3$ and the caprine isolates, respectively (Figure 2). In agreement with those results, rpoB and sod $A$ sequences, respectively, were $100 \%$ identical for both isolates from goats and $100 \%$ identical for isolates from horses $\mathrm{CV} / 2$ and $\mathrm{CV} / 6$ but different between goats and horses and between horse $\mathrm{CV} / 3$ and the other horses. Partial coding sequence data were entered into GenBank under accession numbers EU784814 $(\operatorname{sod} A$, caprine isolates), EU784815 (sodA, equine isolate CV $/ 2$ and $\mathrm{CV} / 6$ ), EU784816 ( $\operatorname{sod} A$, equine isolate $\mathrm{CV} / 3$ ), EU784817 (rpoB, caprine isolates), EU784818 (rрoB equine isolate $\mathrm{CV} / 2$ and $\mathrm{CV} / 6$ ), and EU784819 (rpoB equine isolate $\mathrm{CV} / 3$ ). In agreement with results from sequence-based analysis, the clinical SEZ isolates from the 2 goats showed identical band patterns in RAPDPCR analysis, as did 2 equine isolates, but patterns differed between isolates from goats and horses and between equine isolates $\mathrm{CV} / 2$ and $\mathrm{CV} / 6$ on the one hand and isolate $\mathrm{CV} / 3$ on the other hand (Figure 3 ). Isolates from goat milk were subsequently compared 
Table 2. Classification of 16S-23S intergenic spacer regions (R) of Streptococcus equi ssp. zooepidemicus isolates from goat milk and equine feces based on Chanter et al. (1997) and Hassan et al. (2003)

\begin{tabular}{|c|c|c|c|c|c|c|c|c|c|c|}
\hline Isolate & $\mathrm{R} 1$ & $\mathrm{R} 2$ & $\mathrm{R} 3$ & $\mathrm{R} 4$ & $\mathrm{R} 5$ & $\mathrm{R} 6$ & $\mathrm{R} 7$ & $\mathrm{R} 8$ & $\mathrm{R} 9$ & Reference \\
\hline 922 & $1 \mathrm{a}$ & $27 \mathrm{bp}$ & Absent & $73 \mathrm{bp}$ & $5 \mathrm{~b}$ & $6 \mathrm{~b}$ & $7 \mathrm{c}$ & $90 \mathrm{bp}$ & $19 \mathrm{bp}$ & This study \\
\hline 928 & $1 \mathrm{a}$ & $27 \mathrm{bp}$ & Absent & $73 \mathrm{bp}$ & $5 b$ & $6 \mathrm{~b}$ & $7 \mathrm{c}$ & $90 \mathrm{bp}$ & $19 \mathrm{bp}$ & This study \\
\hline $\mathrm{CV} / 2$ & $1 \mathrm{a}$ & $27 \mathrm{bp}$ & Absent & $73 \mathrm{bp}$ & $5 \mathrm{a}$ & $6 \mathrm{a}$ & $7 \mathrm{~b}$ & $92 \mathrm{bp}$ & $22 \mathrm{bp}$ & This study \\
\hline $\mathrm{CV} / 3$ & $1 \mathrm{a}$ & $27 \mathrm{bp}$ & Absent & $73 \mathrm{bp}$ & $5 \mathrm{a}$ & $6 \mathrm{a}$ & $7 \mathrm{a}$ & $90 \mathrm{bp}$ & 19 bp & This study \\
\hline $\mathrm{CV} / 6$ & $1 \mathrm{a}$ & $27 \mathrm{bp}$ & Absent & $73 \mathrm{bp}$ & $5 \mathrm{a}$ & $6 a$ & $7 \mathrm{~b}$ & $92 \mathrm{bp}$ & $22 \mathrm{bp}$ & This study \\
\hline SEE & $1 \mathrm{a}$ & $27 \mathrm{bp}$ & Present (169 bp) & $73 \mathrm{bp}$ & $5 \mathrm{~b}$ & $6 \mathrm{~b}$ & $7 \mathrm{c}$ & $90 \mathrm{bp}$ & $19 \mathrm{bp}$ & Chanter et al., 1997 \\
\hline SEE AF489598 & $1 \mathrm{a}$ & $27 \mathrm{bp}$ & Present (167 bp) & $73 \mathrm{bp}$ & $5 \mathrm{~b}$ & $6 \mathrm{~b}$ & $7 \mathrm{c}$ & $87 \mathrm{bp}$ & $19 \mathrm{bp}$ & Hassan et al., 2003 \\
\hline SEZ 2809 & $1 \mathrm{a}$ & $27 \mathrm{bp}$ & Absent & $73 \mathrm{bp}$ & $5 \mathrm{a}$ & $6 \mathrm{a}$ & $7 \mathrm{a}$ & $90 \mathrm{bp}$ & $19 \mathrm{bp}$ & Chanter et al., 1997 \\
\hline SEZ K3 & $1 b$ & $27 \mathrm{bp}$ & Present (169 bp) & $73 \mathrm{bp}$ & $5 \mathrm{~b}$ & $6 \mathrm{~b}$ & $7 \mathrm{c}$ & $92 \mathrm{bp}$ & $22 \mathrm{bp}$ & Chanter et al., 1997 \\
\hline SEZ AF489599 & $1 b$ & $27 \mathrm{bp}$ & Absent & $73 \mathrm{bp}$ & $5 \mathrm{a}$ & $6 a$ & $7 \mathrm{a}$ & $90 \mathrm{bp}$ & $22 \mathrm{bp}$ & Hassan et al., 2003 \\
\hline
\end{tabular}

${ }^{1}$ The letters $\mathrm{a}, \mathrm{b}$, and $\mathrm{c}$ are part of the standard nomenclature for classification of the $16 \mathrm{~S}-23 \mathrm{~S}$ intergenic spacer regions.

with fecal isolates obtained from individual horses over time. Multiple banding patterns were observed among fecal isolates from a single horse. Some patterns were observed for isolates obtained on different occasions (Figure 4), but none of the patterns from fecal isolates matched the banding pattern of the caprine isolates.

\section{DISCUSSION}

Approximately $4.7 \%$ of culture-positive cases of clinical mastitis in small ruminants were attributed to SEZ (Las Heras et al., 2002). Although the average number of cases of mastitis due to this organism is low, it can be a serious economic problem in individual herds. The SEZ mastitis described in this work was characterized by a decrease in milk production and high SCC and led to culling of close to $10 \%$ of the herd. Slaughter of infected goats was driven by the effect of the infection on milk quality and production, and the risk of zoonotic SEZ infections associated with the consumption of nonpasteurized milk products (Edwards et al., 1988; Bordes-Benítez et al., 2006; Kuusi et al., 2006). Goat milk and goat milk products can make an important contribution to human nutrition, and consumption of

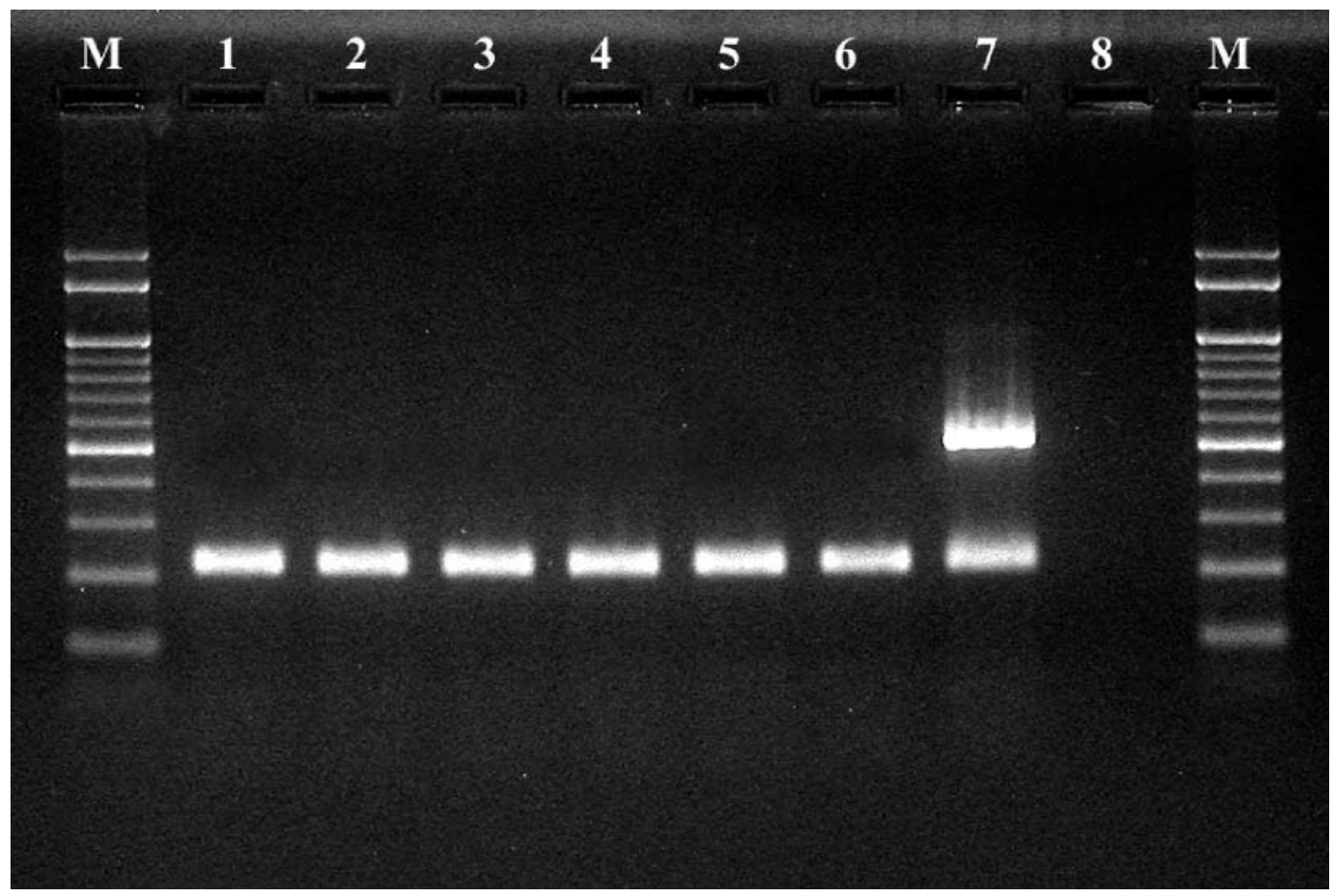

Figure 1. Multiplex PCR amplification of $\operatorname{sodA}(235 \mathrm{bp})$ and seeI (520 bp) genes from streptococcal isolates from goats and horses. Lanes 1 and $2=$ isolates from milk of 2 goats (922 and 928); lanes 3,4 , and $5=$ isolates from feces of 3 horses $(\mathrm{CV} / 2, \mathrm{CV} / 3, \mathrm{CV} / 6) ;$ lane 6 and $7=$ Streptococcus equi ssp. zooepidemicus and S. equi ssp. equi reference strains (ATCC 700400 and ATCC 33398, respectively); lane $8=$ negative control; lane $\mathrm{M}=100-\mathrm{bp}$ DNA ladder. 
ISR922 : AAGGAAAAAGGAAGCACGTTTAGC---GTCTTATTTAGTTTTGAGAGGTCTTGT---GGGGCCTTAGCTCAGCTGGGAGAGCGCCTGCTTTG : 86 ISR928: AAGGAAAAAGGAAGCACGTTTAGC-a--GTCTTATTTAGTTTTGAGAGGTCTTGT---GGGGCCTTAGCTCAGCTGGGAGAGCGCCTGCTTTG : 86 ISRCV/2 : AAGGAAAACGGAAGCACGTTTAGCa---GTCTTATTTAGTTTTGAGAGGTCTTGT---GGGGCCTTAGCTCAGCTGGGAGAGCGCCTGCTTTG : 86 ISRCV/3 : AAGGAAAAAGGAAGCACGTTTAGC---GTCTTATTTAGTTTTGAGAGGTCTTGT---GGGGCCTTAGCTCAGCTGGGAGAGCGCCTGCTTTG : 86 ISRCV/ 6 : AAGGAAAACGGAAGCACGTTTAGC---GTCTTATTTAGTTTTGAGAGGTCTTGT---GGGGCCTTAGCTCAGCTGGGAGAGCGCCTGCTTTG : 86

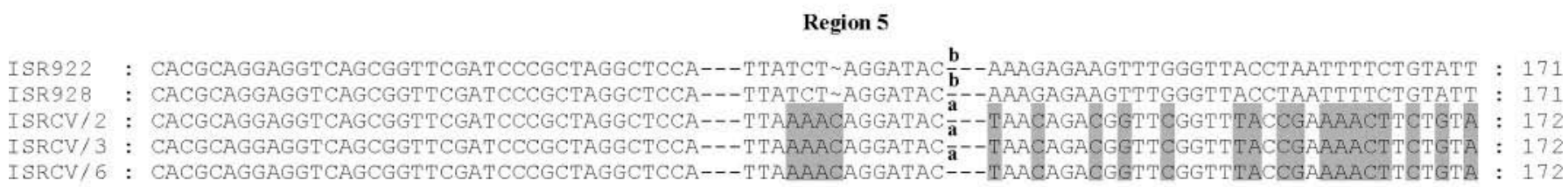

Region 6

ISR922 : CTAAGATATATAAGATATAGTTCAAGTAATTGAACACGACCTCATTTTTTAGGAAAAAAGATAAATCTCCTCGTGTGTAAAGCACACATCGT : 263 ISR928: CTAAGATATATAAGATATAGTTCAAGTAATTGA.ACACGACCTCATTTTTTAGGAAAAAAGATAAATCTCCTCGTGTGTAAAGCACACATCGT : 263 ISRCV/2 : GAAAAATAGGAAACGAGGCTGTGTGTTTAGCACACAAGG AGATTTATCTTTTTTCCTAAGAAGTTAGTCTGGTGTTCAACTTTGTAGATC : 263 ISRCV/3 : GAAAAATAGGAAATCGACGCTGTGTGTTTAGCACACAAGG AGATTTATCTTTTTTCCTAAGAAGTTAGTCTGGTGTTCAACTTTGTAGATC : 263 ISRCV/6 : GAAAAATAGGAAATCGACGCTGTGTGTTTAGCACACAAGG AGATTTATCTTTTTTCCTAAGAAGTIAGTCTGGTGTTCAACTTTGTAGATC : 263

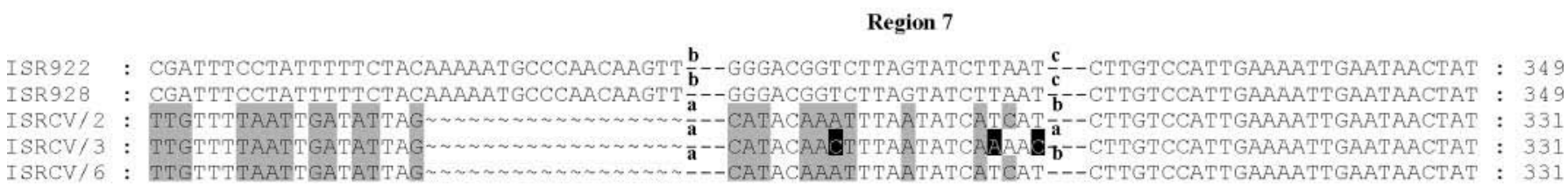

Region 8

Region 9

ISR922 : ATCACATTCCGCAATCAAAAAA GATTGTAAGAAAGTAACAAGAAATAAACCGAAACGCTGTGA---TAACGAGTTTAAAAAA TAA : 431 ISR928: ATCACATTCCGCAATCAAAAAA GATTGTAAGAAAGTAACAAGAAATAAACCGAAACGCTGTGA- - TAACGAGTTTAAAAAA TAA : 431 ISRCV/2 : ATCACATTCCGCAATCAAAAAGAAGATTGTAAGAAAGTAACAAGAAATAAACCGAAACGCTGTGA---TAACGAGTTTAAGAAAAAATAA : 418 ISRCV/3 : ATCAMATCCGCAATCAAAAAA GATTGTAAGAAAGTAACAAGAAATAAACCGAAACGCTGTGA---TAACGAGTTTAAAAAA TAA: 413 ISRCV/6 : ATCACATTCCGCAATCARAAAGAAGATTGTAAGAAAGTAACAAGAAATAAACCGAAACGCTGTGA---TAACGAGTTTAAGAAAAAATAA : 418

Figure 2. Multiple alignment of the 16S-23S intergenic spacer region for isolates from milk of goats (922 and 928) and feces from horses $(\mathrm{CV} / 2, \mathrm{CV} / 3, \mathrm{CV} / 6)$ and definition of 9 regions according to Chanter et al. (1997) and Hassan et al. (2003).

goat milk is increasing (Haenlein, 2004; Dubeuf, 2005), underscoring the importance of mastitis control in goats, particularly mastitis caused by zoonotic pathogens.

Previous studies (Sharp et al., 1995; Bezek, 1998; Las Heras et al., 2002) reported outbreaks of clinical mastitis in cows and small ruminants due to SEZ. The role of horses or donkeys was suspected, but no specific investigation was done to corroborate this point. On the farm involved in this outbreak, 3 horses grazing with the affected goats tested positive for SEZ in feces. The horses were apparently healthy, with no symptoms of respiratory illness, and SEZ could be considered a normal commensal of mucous membranes, especially in the intestines. Identification of hypothetical routes of transmission from horses to goats, whether direct or indirect, can be extremely difficult to achieve. Direct contact constituted physical contact or sufficiently close proximity between 2 animals for transmission of pathogens to occur. Indirect contact involved exposure to contaminated material from infected hosts in the environment, including contact with horse feces, urine, nasal discharge or exudates on grazing land, or within farmyards and buildings. Most goats generally avoid grazing land areas contaminated with feces, but some goats prefer grazing such areas. Moreover, food and water provided for goats on pasture, whether in troughs, on the ground, or from natural water bodies may potentially be contaminated by feces or nasal discharge from horses. Horses on the outbreak farm shared pastures with the goats during grazing. At night, all animals were housed in the same barn. Thus, direct or indirect contact of horses with the goats involved in the outbreak may have resulted in initial exposure and infection of 1 or both goats. The 2 isolates from goats had identical V2, ISR, rро $B$ and $\operatorname{sod} A$ sequences, and RAPD patterns, indicating that both cases of mastitis were produced by a single strain. This could be the result of contagious transmission, or of exposure to a common source in the environment. Decreased milking hygiene may have contributed to the transmission of SEZ among goats. Host-species jumping by Streptococcus species from a primary to a secondary host species, 


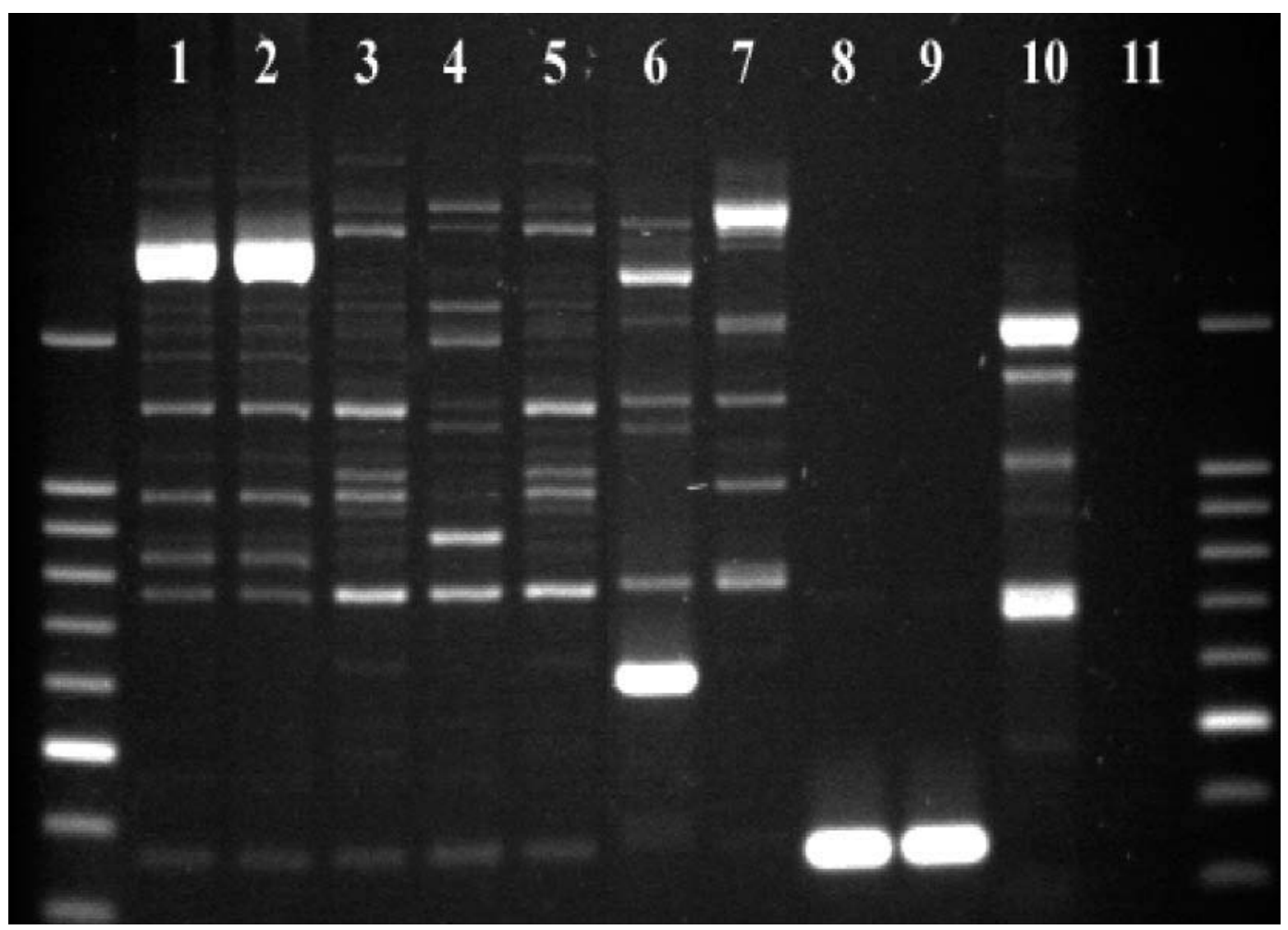

Figure 3. Random amplified polymorphic DNA-PCR banding patterns of Streptococcus equi ssp. zooepidemicus isolates from goats involved in the clinical mastitis outbreak and from horses. Lanes 1 and $2=$ isolates from milk of goats 922 and 928 ; lanes 3 , 4, and $5=$ isolates from feces of 3 horses $(\mathrm{CV} / 2, \mathrm{CV} / 3, \mathrm{CV} / 6)$ collected during the outbreak; lane 6 and $7=S$. equi ssp. equi and S. equi ssp. zooepidemicus reference strains (ATCC 33398 and ATCC 700400, respectively); lanes 8 and $9=$ Staphylococcus aureus (ATCC 29213); lane 10 = Klebsiella pneumoniae (ATCC 13883); lane 11 = negative control.

followed by contagious transmission and IMI in the secondary host, was described for Streptococcus canis (Tikofsky and Zadoks, 2005).

Strains isolated from horse feces differed from those obtained from goat milk, so we could not confirm horses as the source of the mastitis outbreak. Still, the horses cannot be ruled out as a source of the infection. For each milk sample, only 1 SEZ isolate was used for molecular typing based on the premise that multiple streptococcal colonies from a quarter with mastitis usually belong to the same strain (Oliver et al., 1998). Fecal samples can contain multiple strains of mastitis-causing streptococcal species (Zadoks et al., 2005b), and multiple colonies were typed for fecal samples. Within and between fecal samples from individual horses, heterogeneous populations of SEZ were detected. The number of isolates per sample may not have been sufficient to identify all strains that were present in the sample (Döpfer et al., 2008). In addition, fecal samples were collected after the mastitis outbreak had been diagnosed, and fecal strains that were present before the mastitis outbreak may have gone undetected at other time points because shedding of streptococci can be intermittent (Zadoks et al., 2005b). Ideally, an order of events can be established to support the proposed direction of transmission. For streptococci that cause clinical symptoms in both host species, this may be feasible (Tikofsky and Zadoks, 2005). If host species can act as asymptomatic carriers, a timeline may be difficult to establish.

Presence of SEZ in the nose of horses was not detected using culture of nasal swabs. Polymerase chain reaction-based detection of SEZ DNA was more sensitive than bacteriological culture for detection of SEZ in nasal secretions, especially in horses that shed intermittently (Newton et al., 2000). In our study, we needed to obtain viable isolated colonies for comparison of RAPD patterns between equine and caprine isolates. Therefore, PCR was not used as a primary diagnostic method, but only for characterization of isolates. Intermittent nasal shedding of SEZ by horses may have gone undetected.

In summary, horses that shared pasture with goats are the most likely source of the observed outbreak of SEZ mastitis, but an epidemiological relationship could not be confirmed with molecular methods. To improve the methodology of outbreak analysis in future studies, typing of multiple isolates per sample will be necessary, 


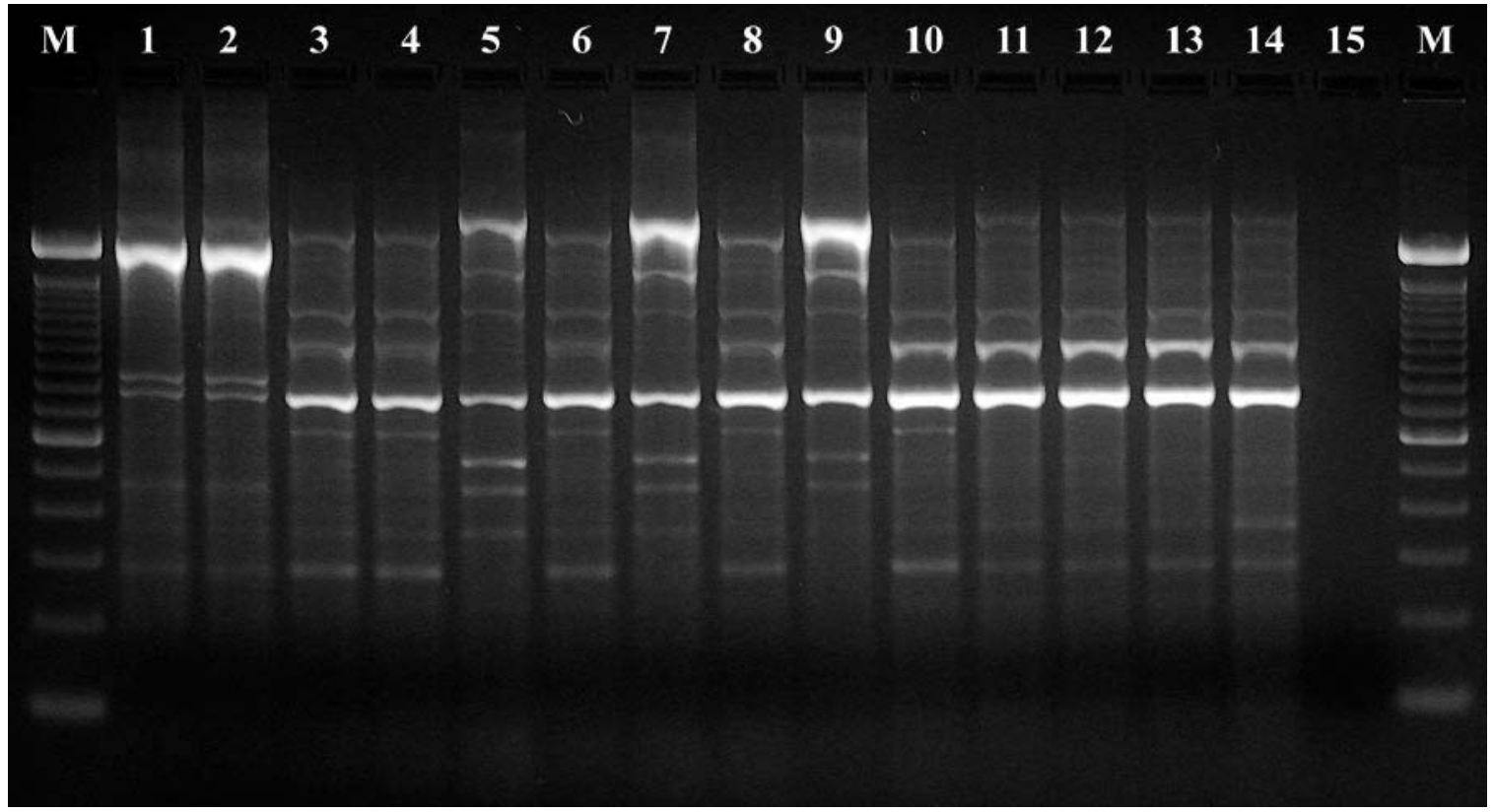

Figure 4. Random amplified polymorphic DNA-PCR banding patterns of 12 Streptococcus equi ssp. zooepidemicus isolates from different fecal samples of the same horse $(\mathrm{CV} / 3)$ on a goat farm. Lanes 1 and $2=$ isolates from milk of goats 922 and 928 , respectively; lanes 3 to $14=$ isolates from the horse at different time points (lanes 3 to $6=$ isolates from fecal sampling after 1 wk; lanes 7 to $10=$ isolates after 1 mo; lanes 11 to $14=$ isolates after $6 \mathrm{mo}$ ); lane $15=$ negative control. Three patterns are distinguished for equine fecal isolates (group $1=$ lanes $3,4,6,8$, 10; group $2=$ lanes 5, 7, 9; and group $3=$ lanes 11, 12, 13, 14), all of which are distinct from the pattern for caprine milk isolates.

especially for sample types that may contain a heterogeneous bacterial population.

\section{REFERENCES}

Abdulmawjood, A., and C. H. Lämmler. 2000. Determination of intraspecies variations of the V2 region of the $16 \mathrm{~S}$ rRNA gene of Streptococcus equi subsp. zooepidemicus. Res. Vet. Sci. 68:33-39.

Alber, J., A. El-Sayed, C. Lämmler, A. A. Hassan, R. Weiss, and M. Zschöck. 2004. Multiplex polymerase chain reaction for identification and differentiation of Streptococcus equi subsp. zooepidemicus and Streptococcus equi subsp. equi. J. Vet. Med. B 51:455-458.

Balter, S., A. Benin, S. W. Pinto, L. M. Teixeira, G. G. Alvim, D. Luna Jackson, L. Laclaire, J. Elliot, R. Facklam, and A. Schuchat. 2000. Epidemic nephritis in Nova Serrana, Brazil. Lancet 355:17761780

Barnham, M., T. J. Thornton, and K. Lange. 1983. Nephritis caused by Streptococcus zooepidemicus (Lancefield group C). Lancet 1:945-948.

Baverud, V., S. K. Johansson, and A. Aspan. 2007. Real-time PCR for detection and differentiation of Streptococcus equi subsp. equi and Streptococcus equi subsp. zooepidemicus. Vet. Microbiol. 124:219229.

Bezek, D. M. 1998. Genus identification and antibiotic susceptibility patterns of bacterial isolates from cows with acute mastitis in a practice population. J. Am. Vet. Med. Assoc. 212:404-406.

Bordes-Benítez, A., M. Sánchez-Oñoro, P. Suárez-Bordón, A. J García-Rojas, J. A. Saéz-Nieto, A. González-García, I. AlamoAntúnez, A. Sánchez-Maroto, and M. Bolaños-Rivero. 2006. Outbreak of Streptococcus equi subsp. zooepidemicus infections on the island of Gran Canaria associated with the consumption of inadequately pasteurized cheese. Eur. J. Clin. Microbiol. Infect. Dis. $25: 242-246$.
Chanter, N., N. Collin, N. Holmes, M. Binns, and J. Mumford. 1997 Characterization of the Lancefield group C streptococcus 16S-23S RNA gene intergenic spacer and its potential for identification and sub-specific typing. Epidemiol. Infect. 118:125-135.

Döpfer, D., W. Buist, Y. Soyer, M. A. Munoz, R. N. Zadoks, L. Geue, and B. Engel. 2008. Assessing genetic heterogeneity within bacterial species isolated from gastrointestinal and environmental samples: How many isolates does it take? Appl. Environ. Microbiol. 74:3490-3496.

Drancourt, M., V. Roux, P. E. Fournier, and D. Raoult. 2004. rpoB gene sequence-based identification of aerobic gram-positive cocci of the genera Streptococcus, Enterococcus, Gemella, Abiotrophia, and Granulicatella. J. Clin. Microbiol. 42:497-504.

Dubeuf, J.-P. 2005. Structural, market and organisational conditions for developing goat dairy production systems. Small Rumin. Res. 60:67-74.

Edwards, A. T., M. Roulson, and M. J. Ironside. 1988. A milk-borne outbreak of serious infection due to Streptococcus zooepidemicus (Lancefield group C). Epidemiol. Infect. 101:43-51.

Haenlein, G. F. W. 2004. Goat milk in human nutrition. Small Rumin. Res. 51:155-163.

Harrington, D., I. Sutcliffe, and N. Chanter. 2002. The molecular basis of Streptococcus equi infection and disease. Microbes Infect. 4:501-510

Hassan, A. A., I. U. Khan, A. Abdulmawjood, and C. Lämmler. 2003. Inter- and intraspecies variations of the $16 \mathrm{~S}-23 \mathrm{~S}$ rDNA intergenic spacer region of various streptococcal species. Syst. Appl. Microbiol. 26:97-103.

Kuusi, M., E. Lahti, A. Virolainen, M. Hatakka, R. Vuento, L. Rantala, J. Vuopio-Varkila, E. Seuna, M. Karppelin, M. Hakkinen, J. Takkinen, V. Gindonis, K. Siponen, and K. Huotari. 2006. An outbreak of Streptococcus equi subspecies zooepidemicus associated with consumption of fresh goat cheese. BMC Infect. Dis. 6:3641.

Las Heras, A., A. I. Vela, E. Fernández, E. Legaz, L. Domínguez, and J. F. Fernández-Garayzábal. 2002. Unusual outbreak of 
clinical mastitis in dairy sheep caused by Streptococcus equi subsp. zooepidemicus. J. Clin. Microbiol. 40:1106-1108.

National Mastitis Council. 1999. Laboratory Handbook on Bovine Mastitis. National Mastitis Council, Madison,WI.

Newton, J. R., K. Verheyen, N. C. Talbot, J. F. Timoney, J. L. Wood, K. H. Lahani, and N. Chanter. 2000. Control of strangles outbreaks by isolation of guttural pouch carriers identified using PCR and culture of Streptococcus equi. Equine Vet. J. 32:515-526.

Oliver, S. P., B. E. Gillespie, and B. M. Jayarao. 1998. Detection of new and persistent Streptococcus uberis and Streptococcus dysgalactiae intramammary infections by polymerase chain reaction-based DNA fingerprinting. FEMS Microbiol. Lett. 160:69-73.

Sharp, M. W., M. J. Prince, and J. Gibbens. 1995. S. zooepidemicus infection and bovine mastitis. Vet. Rec. 137:128. PubMed

Tikofsky, L. L., and R. N. Zadoks. 2005. Cross-infection between cats and cows: Origin and control of Streptococcus canis mastitis in a dairy herd. J. Dairy Sci. 88:2707-2713.

Timoney, J. F. 2004. The pathogenic equine streptococci. Vet. Res. 35:397-409.
Vogel, L., G. Jones, S. Triep, A. Koek, and L. Dijkshoorn. 1999. RAPD typing of Klebsiella pneumoniae, Klebsiella oxytoca, Serratia marcescens and Pseudomonas aeruginosa isolates using standardized reagents. Clin. Microbiol. Infect. 5:270-276.

Younan, M., A. T. Estoepangestie, M. Cengiz, J. Alber, A. El-Sayed, and C. Lämmler. 2005. Identification and molecular characterization of Streptococcus equi subsp. zooepidemicus isolated from camels (Camelus dromedarius) and camel milk in Kenya and Somalia. J. Vet. Med. B 52:142-146.

Zadoks, R. N., Y. H. Schukken, and M. Wiedmann. 2005a. Multilocus sequence typing of Streptococcus uberis provides sensitive and epidemiologically relevant subtype information and reveals positive selection in the virulence gene pauA. J. Clin. Microbiol. 43:2407-2417.

Zadoks, R. N., L. L. Tikofsky, and K. J. Boor. 2005b. Ribotyping of Streptococcus uberis from a dairy's environment, bovine feces and milk. Vet. Microbiol. 109:257-265. 